\title{
Enhancement of Performance Parameters of Printed E- Shaped Slot and Microstrip Patch Antennas using CPW
}

\author{
Swarnima Sharma \\ DIT University
}

\author{
Dhruva Chaudhary \\ Asst. Professor \\ DIT University
}

\author{
Gurjeet Kaur \\ DIT University
}

\begin{abstract}
Two printed wide-slot antennas with E-shaped patches and slots for broadband applications are proposed. They are fed by a coplanar waveguide (CPW) and a Microstrip line with the performances that are almost same. Detailed simulation and experimental investigations are conducted to understand their behaviour and optimize for operations of broadband. Good agreement between the measurement and simulation has been achieved. We have obtained the large operating bandwidth by choosing suitable combinations of feed and shapes of slot. In order to achieve wider operation bandwidth both of the designed antennas have round corners on the wide patch and slot. Meanwhile the proposed antennas exhibit almost omnidirectional radiation patterns and low cross polarization and relatively high gain. A comprehensive numerical sensitivity analysis has been done to understand the effects of various dimensional parameters and to optimize the performance of the designed antennas.
\end{abstract}

\section{Keywords}

Bandwidth, E-shaped slot antennas, Microstrip Antenna, coplanar waveguide (CPW).

\section{INTRODUCTION}

Microstrip patch antennas (MPAs) are widely used in today's era. They are attractive for their well known efficient features such as compatability with monolithic microwave integrated circuits. They are used in different fields like broadband direction finder systems, satellite and communication applications due to its compact shape and light weight, less complexity and ease of implementation[1]. Despite of these advantages Microstrip patch antennas have some drawbacks as well such as low impedance bandwidth and low gain. To overcome the drawbacks of the common Microstrip antennas and slot antennas are currently in consideration for the use in broadband communication systems as they provide wide bandwidths, good impedance matching and bidirectional or unidirectional radiation patterns[2].

In this communication, two printed wide-slot antennas with Eshaped patches and slots for broadband communication systems are presented. They are fed by a coplanar waveguide (CPW) and a Microstrip line with almost the same performances. The antenna fed by the Microstrip line has a bandwidth from $2.85 \mathrm{GHz}$ to $15.12 \mathrm{GHz}$ and the antenna fed by the coplanar waveguide (CPW) line has the bandwidth from $2.83 \mathrm{GHz}$ to $18.2 \mathrm{GHz}[3]$. The proposed slot antennas have wider operation bandwidths and higher gains and lower cross polarisations and much smaller sizes. In order to achieve wider operation bandwidth both of the Microstrip line fed and coplanar waveguide line fed antennas have four round corners on the wide slot and patch. The bandwidths of the proposed antennas are largely enhanced by rounding the end portions of the branches of the E-shaped slots and feed patches[4].
This combination is selected because of its broadband characteristics with almost omnidirectional radiation patterns over the entire bandwidth. The used feeding techniques have almost the same performance but are different in their designs. For the Microstrip line fed antenna and the slot and the feeding line are printed on different sides of the dielectric substrate. For the CPW fed antenna and the slot and the feeding line are printed on the same side of the substrate[5]. Moreover this difference in designing is just to overcome the problem occurring in the Microstrip line fed antenna. In the Microstrip line fed antenna and misalignment can result because etching is required on both sides of the dielectric substrate. This alignment error is eliminated by using a CPW line fed antenna as it is used to excite the slot since etching of the slot and the feeding line is one sided[6].

The proposed work shows that large operating bandwidth is obtained by choosing suitable combinations of feed and slot shapes. Moreover, it will be clear that by rounding the end portions of the branches of the E-shaped slots and feed patches, the bandwidths of the antennas are largely enhanced. In addition to be small in size then the proposed antennas exhibit stable and almost omnidirectional radiation patterns in the entire operating bandwidth, relatively high gains and low cross polarisation[7].

\subsection{Design with an E-Shaped Patch}

Similar broadband operation can be obtained by using an Eshaped patch. The E-shaped patch is formed by inserting a pair of wide slits at the boundary of a Microstrip patch.

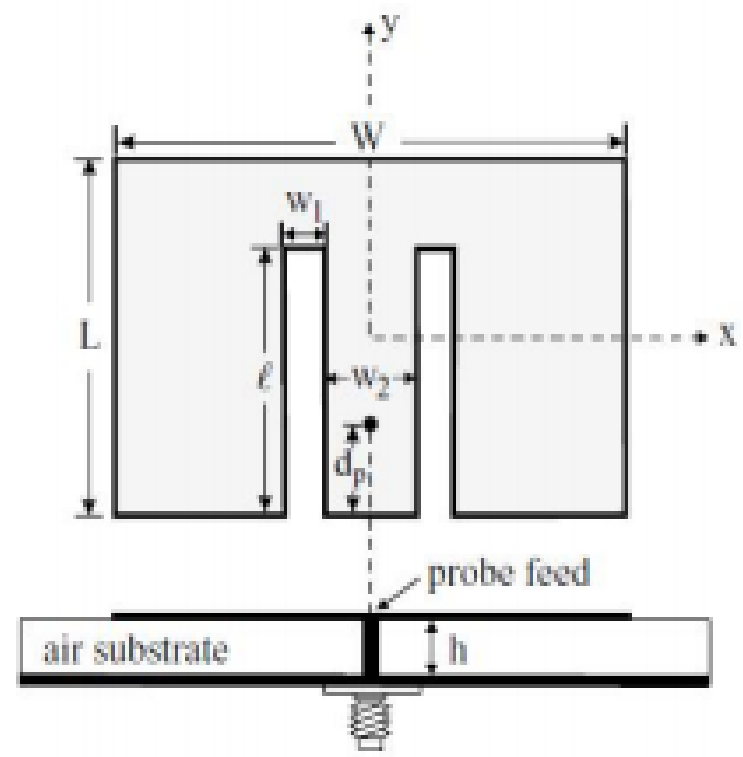

Figure 1. Geometry of a broadband E-shaped Microstrip antenna 
Non conducting posts of height $h$ (not shown in the figure) from the ground plane. The two wide slits have the same length 1 and the same width w1 and are inserted at the bottom edge. The separation of the two wide slits is w2 and the two slits are placed symmetrically with respect to the patch's center line (y axis). There are thus only three parameters (l, w1, w2) for the wide slits used. Along the patch's center line a probe feed at a distance dp from the patch's bottom edge can be located for good excitation of the proposed antenna over a wide bandwidth[8].

\section{MICROSTRIP ANTENNA}

A Microstrip patch antenna consists of a radiating conducting strip placed on a grounded dielectric layer. Design of the radiating patch (length, width, feed type etc.) and characteristic of the dielectric substrate (dielectric constant, height of the substrate etc.) determines the behaviour of the antenna. The center conductor of a coaxial cable serves as the feed probe to couple electromagnetic energy in and/or out of the patch [4]. The electric field distribution of a rectangular patch excited in its fundamental mode is also indicated. Microstrip patch can be of different shapes such as rectangular, square or disk patches. They can provide linear, dual or circular polarization by appropriate feeding. Patch antennas are low cost have a low profile and are easily fabricated[16].

Consider the Microstrip antenna shown in Figure fed by a Microstrip transmission line. The patch antenna and Microstrip transmission line and ground plane are made of high conductivity metal (typically copper). The patch is of length $\mathrm{L}$ and width $\mathrm{W}$ and sitting on top of a substrate of thickness h with permittivity $\mathcal{E}$. The thickness of the ground plane. Typically the height $h$ is much smaller than the wavelength of operation but not much smaller than 0.05 of a wavelength[9].The frequency of center will be approximately given by:

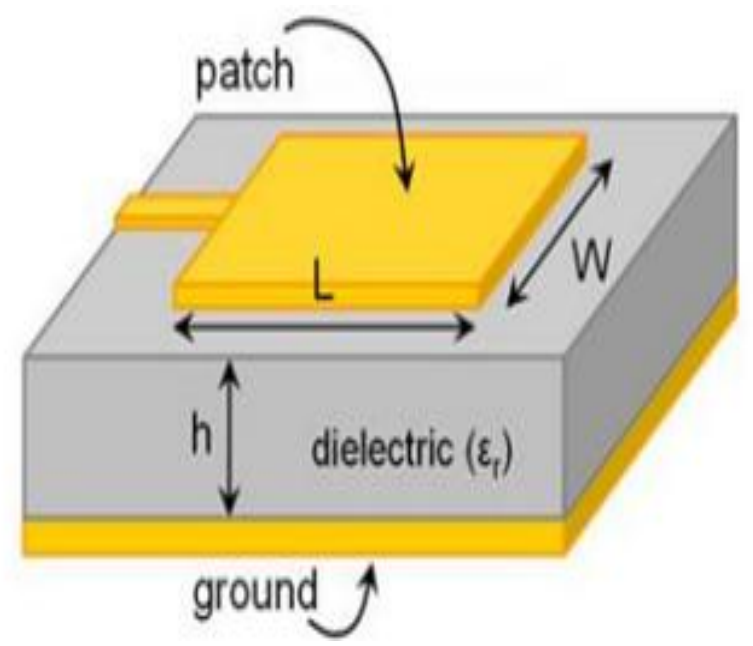

Figure2. Typical Microstrip Patch Antenna

The electric field is zero at the center of the patch and maximum (positive) at one side and minimum (negative) on the opposite side. The minimum and maximum electric fields continuously change side according to the instantaneous phase of the applied signal. The electric field shown above does not stop abruptly at the patch's periphery as in cavity but the fields extend the outer periphery to some degree. These fields extension are known as fringing fields and cause the patch to radiate[10].
The dielectric substrate act as an electrical insulator is a substance that is highly resistant to the flow of electric current. Ideally, the dielectric constant $\mathcal{E}_{\mathrm{r}}$ of the substrate should be low $(\varepsilon \mathrm{r}<2.5)$ to enhance the fringing field that account for the radiation. However in some cases, other performance requirements may dictate the use of dielectric material whose dielectric constants can be greater for $\mathcal{E} r>4$ [11].

\subsection{Applications of Microstrip Antenna}

The wide range of application from transportation, communication to biomedical can be seen [13].

- Aircraft and ship antennas: - Communication and navigation, altimeters, blind landing Systems.

- Missiles:- Radar, proximity fuses and telemetry

- $\quad$ Satellite communications:- Domestic direct broadcast $\mathrm{TV}$, vehicle-based antennas, communication

- Mobile:- radio Pagers and hand phones, man pack systems, mobile vehicle

- Remote sensing:- Large, lightweight apertures

- Biomedical Applicators:- in microwave hyperthermia

- Others:- Intruder alarms, personal communication etc[19].

\section{COPLANAR WAVEGUIDE}

Coplanar waveguide is type of electrical transmission line which can be fabricated using printed circuit board technology and is used to convey microwave-frequency signals. On the smaller scale, coplanar waveguide transmission lines are also built into the monolithic microwave integrated circuits[12]. Conventional coplanar waveguide (CPW) consists of a single conducting track printed onto a dielectric substrate together with a pair of return conductors one to either side of the track. All of three conductors are on the same side of the substrate and hence are coplanar. The return conductors are separated and different from the central track by a small gap, which has been an unvarying width along the length of line. Apart from this the central conductor the return conductors usually extended to an indefinite but large distance so the each is notionally a semi-infinite plane[14].

\section{PREVIOUS WORK}

Various research papers related to Microstrip patch antennas (MPAs) are studied and discussed in this section. Microstrip patch antennas (MPAs) are widely used in today's era. They are attractive for their well known efficient features such as compatability with monolithic microwave integrated circuits. They are used in different fields like broadband direction finder systems, satellite and communication applications due to its compact shape and light weight, less complexity and ease of implementation[15]. Despite of these advantages Microstrip patch antennas have some drawbacks as well such as low impedance bandwidth and low gain. To overcome the drawbacks of the common Microstrip antennas, slot antennas are currently in consideration for the use in broadband communication systems as they provide wide bandwidths, good impedance matching, and bidirectional or unidirectional radiation patterns[17]. 


\subsection{J.-Y. Sze and K.-L. Wong, "Bandwidth enhancement of a Microstrip line-fed printed wide-slot antenna,"'[1]}

The study of a new design of Microstrip line-fed printed wideslot antennas with a fork-like tuning stub for bandwidth enhancement was reported and the radiation characteristics of such a design were investigated. The fork-like tuning stub studied there, was all positioned within the slot region in the opposite side of the printed wide slot. Through proper selection of the parameters of the fork-like tuning stub, it was expected that the coupling between the Microstrip line and the printed wide slot can be controlled more effectively, which made significant bandwidth enhancement possible of the printed wide-slot antenna. The conclusion here was drawn after observing the impedance characteristics and radiation characteristics of the proposed antenna. It was observed that the bandwidth enhancement is obtained mainly through the enhanced coupling between the Microstrip feed line and the printed wide slot by the fork-like tuning stub. And for the frequencies within the impedance bandwidth larger than $2400 \mathrm{MHz}$, the radiation patterns were found to be titled to a large angle and the maximum radiation direction was no longer in the broadside direction of the slot antenna. The problem that was observed in this proposed antenna was that for higher frequencies in the impedance bandwidth, some undesired higher order modes of the printed wide sot antenna are also excited, which caused some distortions in the resultant radiation patterns.

\subsection{H. L. Lee, H. J. Lee, J. G. Yook, and H. K. Park, "Broadband planar antenna having round corner rectangular wide slot,",[2]}

In this paper, it was proved that more wide imedance bandwidth can be achieved by using round corner rectangular slot. Various shapes of Microstrip feed line with rectangular wide slot were reported for wideband antennas application and thus a novel Microstrip feed line structure and round corner wide slot antennas that achieved a good impedance match over a wide operating frequency range was presented. This was done because a round corner rectangular wide slot antenna has more wide operating frequency range than a normal rectangular wide slot antenna. Another reason to choose the round corner rectangular slot was their appearance. It appeared to be a smaller slot size than normal rectangular slot. And it was found that smaller slot size causes improvement in impedance matching at higher end of the frequency band.

\subsection{H.-D. Chen, "Broadband CPW-fed square slot antennas with a widened tuning stub," [3]}

In this piece of work, a square slot antenna for broadband operation was presented by using a coplanar waveguide (CPW) fed with a widened tuning stub. In this piece of work it was observed that the impedance matching strongly depends on the location of the tuning stub in the square slot. By properly choosing the location and size of the tuning stub, wide impedance bandwidth of $60 \%$ can be obtained. For dis proposed antenna it was noted that an impedance bandwidth larger than $50 \%$ can be obtained when the width (w) is chosen in the wide range from $26(=0.59 \mathrm{~L})$ to $40 \mathrm{~mm}(=0.9 \mathrm{~L})$. Therefore, a conclusion was drawn that good impedance was achieved simply by tuning the spacing between the tuning stub and the ground plane.

\section{CONCLUSION}

CPW fed antenna has a larger impedance bandwidth ranging from $2.83 \mathrm{GHz}$ to $18.2 \mathrm{GHz}$. This large operating bandwidth is obtained by choosing suitable combinations of feed and slot shapes. Moreover, by rounding the end portions of the branches of the E-shaped slots and feed patches and the bandwidths of the antennas are largely enhanced[18]. In addition to be small in size, this antenna exhibit stable and almost omnidirectional radiation patterns in the entire operating bandwidth, relatively high gains, and low cross polarisation. After studying these characteristics the conclusion drawn is that the wide-slot antennas can be useful for broadband direction finder systems, satellite and communication applications.

\section{REFERENCES}

[1] J.-Y. Sze and K.-L. Wong, "Bandwidth enhancement of a microstrip line-fed printed wide-slot antenna," IEEE Trans. Antennas Propag.,vol. 49, no. 7, pp. 1020-1024, Jul. 2001.

[2] H. L. Lee, H. J. Lee, J. G. Yook, and H. K. Park, "Broadband planar antenna having round corner rectangular wide slot," in Proc. IEEE Antennas and Propagation Society Int. Symp., Jun. 16-21, 2002, vol. 2, pp. $460-463$.

[3] H.-D. Chen, "Broadband CPW-fed square slot antennas with a widened tuning stub," IEEE Trans. Antennas Propag., vol. 51, no. 4, pp. 1982-1986, Aug. 2003.

[4] J.-S. Row and S.-W. Wu, "Circularly-polarized wide slot antenna loaded with a parasitic patch," IEEE Trans. Antennas Propag., vol. 56, no. 9, pp. 2826-2832, Sep. 2008.

[5] C.-C. Yu and X.-C. Lin, "A wideband single chip inductor-loaded CPW-fed inductive slot antenna," IEEE Trans. Antennas Propag., vol.56, no. 5, pp. 1498-1501, May 2008.

[6] P. H. Rao, "Feed effects on the dimensions of wide-band slot antennas," Microw. Opt. Tech. Lett., vol. 40, pp. $77-$ 79, 2004.

[7] Y. W. Jang, "Experimental study of large bandwidth three-offset micro stripline-fed slot antenna," IEEE Microw. Wireless Comp. Lett., vol. 11, pp. 425-426, 2001.

[8] P. H. Rao, V. F. Fusco, and R. Cahill, "Linearly polarized radial stub fed high performance wide-band slot antenna," Electron Lett., vol. 37, pp. 335-337, 2001.

[9] X.-C. Lin and C.-C. Yu, "A dual-band CPW-fed inductive slot-monopole hybrid antenna," IEEE Trans. Antennas Propag., vol. 56, no. 1, pp. 282-285, Jan. 2008.

[10] J.-S. Chen, "Dual-frequency annular-ring slot antennas fed by CPW feed and Microstrip line feed," IEEE Trans. Antennas Propag., vol. 53, no. 1, pp. 569-573, Jan. 2005.

[11] A. U. Bhobe, C. L. Holloway, M. Piket-May, and R. Hall, "Wide-band slot antennas with CPW feed lines: Hybrid and log-periodic designs," IEEE Trans. Antennas Propag., vol. 52, no. 10, pp. 2545-2554, Oct. 2004.

[12] W.-S. Chen and F.-M. Hsieh, "A broadband design for a printed isosceles triangular slot antenna for wireless 
communications," Microw. J., vol. 48, no. 7, pp. 98-112, 2005

[13] J.-Y. Chiou, J.-Y. Sze, and K.-L.Wong, "A broad-band CPW-fed striploaded square slot antenna," IEEE Trans. Antennas Propag., vol. 51, no. 4, pp. 719-721, Apr. 2003.

[14] W.-S. Chen, "A novel broadband design of a printed rectangular slot antenna for wireless applications," Microw. J., vol. 49, no. 1, pp. 122-130, 2006.

[15] Y.-C. Lin and K.-J. Hung, "Compact ultrawide-band rectangular aperture antenna and band-notched designs," IEEE Trans. Antennas Propag., vol. 54, no. 11, pp. 30753081, Nov. 2006.
[16] T. A. Denidni and M. A. Habib, "Broadband printed CPW-fed circular slot antenna," Electron. Lett. vol. 42, no. 3, pp. 135-136, Feb. 2006.

[17] P. Li, J. Liang, and X. Chen, "Study of printed elliptical/circular slot antennas for ultra wideband applications," IEEE Trans. Antennas Propag., vol. 54, no. 6, pp. 1670-1675, June 2006.

[18] J.-Y. Sze, C.-I. G. Hsu, and S.-C. Hsu, "Design of a compact dual-band annular-ring slot antenna," IEEE Antennas Wireless Propag. Lett., vol.6, pp. 423-426, 2007. 